\title{
Small molecule SIRT1 activators for the treatment of aging and age-related diseases
}

\author{
Basil P. Hubbard ${ }^{1,2}$ and David A. Sinclair ${ }^{1,3}$ \\ ${ }^{1}$ Department of Genetics, Harvard Medical School, Boston, MA, 02115 USA \\ ${ }^{3}$ Department of Pharmacology, School of Medical Sciences, The University of New South Wales, \\ Sydney, NSW, Australia 2052
}

\section{Abstract}

Recent studies in mice have identified single molecules that can delay multiple diseases of aging and extend lifespan. In theory, such molecules could prevent dozens of diseases simultaneously, significantly extending healthy years of life. In this review we discuss recent advances, controversies, opportunities, and challenges surrounding the development of SIRT1 activators, molecules with the potential to delay aging and age-related diseases. Sirtuins comprise a family of $\mathrm{NAD}^{+}$-dependent deacylases that are central to the body's response to diet and exercise. New studies indicate that both natural and synthetic sirtuin activating compounds (STACs) work via a common allosteric mechanism to stimulate sirtuin activity, thereby conferring broad health benefits in rodents, primates, and possibly humans. The fact that the two-thirds of people in the USA who consume multiple dietary supplements consume resveratrol, a SIRT1 activator, underscores the importance of understanding the biochemical mechanism, physiological effects, and safety of STACs.

\section{Keywords}

sirtuin; deacetylase; STAC; allosteric activator; aging; chromatin; diabetes; cancer; cardiovascular disease; inflammation

\section{The quest to delay aging}

History and myth abound with discoveries of potions that impart longer life. Only recently, however, has our level of knowledge reached a point where a panacea - a medicine that prevents and treats multiple age-related diseases - is scientifically possible. This optimism is afforded by the relatively recent discovery of genes and small molecules that can extend lifespan in yeast, worms, flies, and mice [1-5]. There are at least three main pathways that control lifespan in mammals: insulin/IGF-1, TSC/mTOR, and the sirtuins [3, 6, 7]. These pathways are believed to control the response to adversity and cellular stress, such as DNA damage, hypoxia, or a reduced supply of resources from the environment. Molecules that modulate each of these pathways are known and have been shown in animals to prevent a

\section{(C) 2013 Elsevier Ltd. All rights reserved.}

Correspondence: david_sinclair@hms.harvard.edu.

${ }^{2}$ Present address: Department of Chemistry and Chemical Biology, Harvard University, 12 Oxford Street, Cambridge, MA, 02138

Publisher's Disclaimer: This is a PDF file of an unedited manuscript that has been accepted for publication. As a service to our customers we are providing this early version of the manuscript. The manuscript will undergo copyediting, typesetting, and review of the resulting proof before it is published in its final citable form. Please note that during the production process errors may be discovered which could affect the content, and all legal disclaimers that apply to the journal pertain. 
diverse set of age related diseases, including cancer, cardiovascular disease, osteoporosis, and type 2 diabetes [2, 3, 6-8]. Whether drugs can be made to safely modulate these pathways and retard aging in humans is not yet known, but we appear to be closer than ever. In this review, we focus on pharmacological activation of a key sirtuin, SIRT1, an area of research that has undergone considerable progress recently, with paradigms challenged and key questions being resolved.

\section{The Sirtuin longevity pathway}

The silent information regulator (SIR) genes promote longevity in diverse species and mediate many of the beneficial effects of calorie restriction (CR), such as a reduced incidence of cancer, cardiovascular disease and diabetes $[9,10]$. The link between sirtuins and aging was first made in budding yeast: overexpression of the SIR 2 gene increased longevity by suppressing rDNA circle formation, a cause of aging in yeast [11]. Overexpression of Sir2 homologs in worms and flies also extended their lifespan [12, 13]. These results were challenged [14] but recently reaffirmed [15, 16]. In mice, at least two sirtuins can promote lifespan extension: SIRT6 overexpression extends the lifespan of male mice when overexpressed in the whole body [17], and SIRT1 extends the lifespan of mice when overexpressed in the brain [18].

Sir2 and its homologs are collectively known as class III histone deacetylases or "sirtuins," and are distinguished from class I and II deacetylases by their requirement for betanicotinamide adenine dinucleotide $\left(\mathrm{NAD}^{+}\right)$as a co-substrate $[19,20]$. Deacetylation by sirtuins involves the consumption of $\mathrm{NAD}^{+}$and acetylated protein substrate to produce nicotinamide (NAM), 2' O-acetyl-adenosine diphosphate-ribose (O-AcADPR), and deacetylated substrate $[6,19]$. In the first step of the reaction, ADP-ribose is covalently attached to the acetyl moiety of the substrate, accompanied by release of free NAM [6, 19]. Hydrolysis of the acetyl-lysine bond then occurs, liberating O-AcADPR [6, 19]. NAM acts as an inhibitor of the reaction, and thus provides negative feedback inhibition of the sirtuins in vivo $[6,19]$.

In mammals, there are seven sirtuin homologs (SIRT1-7). SIRT1, SIRT6, and SIRT7 localize primarily to the nucleus, SIRT3, SIRT4, and SIRT5, localize to mitochondria, and SIRT2 localizes to the cytosol [6]. Though sirtuins were originally described as deacetylases, it is now evident that they have broader activity [6]. In addition to deacetylation, SIRT5 possesses desuccinylase and demalonylase activities [6], SIRT4 and SIRT6 are mono-ADP-ribosyltransferases [6], and SIRT6 can deacylate long chain fatty acids [21]. Indeed, it has been shown that the ability to catalyze long-chain deacylation is a general feature of mammalian sirtuins, and that in the case of SIRT6, long-chain fatty acids can enhance deacetylase activity [22].

Of all the sirtuins, SIRT1 has received the most attention. It deacetylates key histone residues involved in the regulation of transcription, including H3-K9, H4-K16, and H1-K26, and multiple non-histone protein targets including p53, FOXO1/3, PGC-1a, and NF- $\kappa \mathrm{B}$ [6]. By targeting these proteins, SIRT1 is able to regulate numerous vital signaling pathways, including DNA repair and apoptosis, muscle and fat differentiation, neurogenesis, mitochondrial biogenesis, glucose and insulin homeostasis, hormone secretion, cell stress responses, and circadian rhythms [6]. The other sirtuins also play important roles such as regulating mitochondrial reactions, glucose and insulin homeostasis, hepatic lipogenesis, DNA damage, telomere maintenance, inflammation, and the response to hypoxia [6].

Though it was originally a controversial idea, there is now overwhelming evidence that sirtuins underlie at least some of the salient health benefits of CR [6]. Early studies showed 
that lifespan extension by CR requires Sir2 in S. cerevisiae [23-25] and in flies [13]. In worms, while still controversial [26], multiple groups have demonstrated a partial requirement for SIR2.1 for the longevity effects of CR, depending on the diet [27, 28]. In mammals, SIRT1 proteins levels are induced by both fasting and by $\mathrm{CR}$ in numerous tissues [29], and deletion of SIRT1 prevents some of the behavioral changes and health benefits of CR [6]. Furthermore, whole body SIRT1 transgenic mice display phenotypes resembling CR [30], and mice overexpressing SIRT1 in the brain live longer [6]. Importantly, the involvement of SIRT1 in the CR response appears to be tissue specific, in that deletion of SIRT1 in the liver is dispensable for this effect [31].

Consistent with its central role in the CR response, SIRT1 upegulation counteracts a wide variety of age-related diseases in animal models, including cancer, Alzheimer's disease, and type 2 diabetes [6]. Several studies have demonstrated a protective role for SIRT1 in reducing tumor number and growth in colon cancer, prostate cancer, and DNA-damage induced thymic lymphomas [6]. Likewise, mice overexpressing SIRT1 have greatly increased mitochondrial activity [32] and are protected against tumorigenesis, neurodegeneration, and age-related metabolic decline [6]. Recently, a mutation in the SIRT1 gene was identified in humans that predisposes individuals to increased inflammation and type 1 diabetes [33]. Over the last few years, other sirtuins have been linked to the effects of $\mathrm{CR}$ in mammals [6]. For example, SIRT3 is required for the effects of CR on urea metabolism and the well-established ability of CR to protect mice against age-related hearing loss [6].

\section{Natural and synthetic SIRT1 activating compounds (STACs)}

Given their apparent role in mediating the health benefits of $\mathrm{CR}$, and the subsequent demonstration of therapeutic value in pre-clinical animal models, sirtuins have attracted considerable interest as a drug target [34]. Most studies indicate that the broadest utility would come from molecules that activate one or more of the sirtuins, while inhibition of SIRT1 shows promise in some cancer models as well [6]. Both inhibitors (Box 1) and activators have been discovered for sirtuins; some act specifically, others across the entire family [6].

\section{Box 1}

\section{SIRT1 inhibitors}

Nicotinamide (NAM) inhibits sirtuin activity in vitro and in vivo by binding within the Cpocket and reversing the first catalytic step [19, 91]. Several mechanism-based inhibitors of sirtuins have also been reported, including carba-NAD ${ }^{+}$[92], thioacetyl-lysine peptides [93], and acetylated-lysine-ADP ribose conjugates [94]. High-throughput screening and rational design have resulted in the development of a number of more potent sirtuin inhibitors. For example, sirtinol, splitomycin, and suramin robustly inhibit a number of sirtuin isoforms [95]. SIRT1-specific inhibitors include compounds such as tenovins (SIRT1 and SIRT2) [96], MC2141 [97], and EX-527, an inhibitor with an $\mathrm{IC}_{50}$ in the submicromolar range [98]. The latter compound appears to inhibit SIRT1 by binding to the nicotinamide pocket where it stabilizes the closed enzyme conformation and prevents product release [99]. SIRT1 inhibitors may be of therapeutic use for the treatment of specific diseases including certain cancers [100], and autosomal-dominant polycystic kidney disease [101].

By competing with the endogenous sirtuin inhibitor NAM, the synthetic molecule isonicotinamide (iNAM) can act as a pan-sirtuin activator [35]. However, levels of iNAM must 
reach millimolar concentrations within the cell, limiting the pharmacological value of this approach [35]. The first potent sirtuin activating compounds (STACs) were identified in a high-throughput screen using recombinant human SIRT1 [5]. Several classes of plantderived metabolites such as flavones, stilbenes, chalcones and anthocyanidins were shown to directly activate SIRT1 in vitro through an apparent allosteric mechanism involving the lowering of peptide substrate $K_{\mathrm{m}}$ [5]. Most of the activators identified were polyphenolic with a structure-activity relationship characterized by planar multi-phenyl rings bearing hydroxyl groups (Figure 1) [5]. Resveratrol, the most potent of the natural activators, enhanced SIRT1-mediated deacetylation by eightfold using a biochemical assay we discuss below. While the conservation of this mechanism in lower organisms is debated [26], it appears to be conserved in yeast, flies and worms, with several studies reporting that resveratrol extends lifespan in these models in a Sir2-dependent manner $[1,5,15]$.

Of all the natural SIRT1 activators discovered to date, resveratrol (3,5,4'-trihydroxystilbene) is still the most potent. Resveratrol was initially identified in 1940 as a phenolic substance in the white hellebore, Veratrum grandiflorum, a flowering plant [36], and later in grape vines and the Japanese knotweed Polygonum sachalinense [36]. Resveratrol exists as two geometric isomers, with the trans configuration undergoing isomerisation to the cis- form following exposure to ultraviolet light [37]. Prior to its discovery as a SIRT1 activator, resveratrol and related polyphenolic compounds had been documented to possess many health-enhancing effects [36], ostensibly due to their antioxidant properties. In the 1990's, resveratrol and related molecules in red wine were proposed as an explanation for the 'French paradox', the fact that certain European populations with high wine consumption have low rates of cardiovascular disease despite their fat-rich diets [36] (Box 2).

\section{Box 2}

\section{Resveratrol and the French Paradox}

Whether resveratrol underlies the health benefits associated with wine drinking has been the subject of much contention. Studies employing resveratrol for various therapeutic benefit have utilized a wide-range of concentrations, from $0.1-1500 \mathrm{mg}$ per $\mathrm{kg}$ body weight [36]. Based on typical resveratrol concentration estimates in wine, it has been calculated that a daily intake of two glasses of wine would provide a $70 \mathrm{~kg}$ person with a dose of $\sim 27 \mu \mathrm{g}$ per $\mathrm{kg}$ per day, a dose that falls within this therapeutic window [36]. In contrast, Gresele et al. [102] determined that after 15 days of consumption of $300 \mathrm{~mL}$ of wine (white or red), the total plasma resveratrol concentration, including metabolites, was only slightly increased above background. Another study showed that different dietary regimens and inter-individual variation can drastically affect plasma levels of resveratrol [87]. The authors of this study concluded that a complex mixture of polyphenols, rather than solely resveratrol, likely accounts for the French Paradox [87].

The discovery of natural STACs was a proof of concept that prompted screens for synthetic SIRT1 activators that were more potent, soluble and bioavailable. The first synthetic STACs [8] were derivatives of an imidazothiazole scaffold (e.g. SRT1460, SRT1720, and SRT2183) and chemically distinct from the polyphenol backbone of resveratrol. Molecules such as SRT1720 (Figure 1) were shown to activate SIRT1 via the same $K_{\mathrm{m}}$-lowering mechanism as that of resveratrol but with a much lower $\mathrm{EC}_{50}$, the concentration required to increase activity by 50\% [8]. Extremely potent second generation STACs based on benzimidazole and urea-based scaffolds were subsequently described (Figure 1) [38, 39]. 


\section{Mechanism of sirtuin activation}

Though it is generally accepted that STACs increase SIRT1 activity in vivo, the mechanism by which they activate SIRT1 was the subject of intense debate [40, 41]. Two opposing models were proposed to account for STAC activity: (i) direct allosteric activation of Sir2/ SIRT1 through a lowering of peptide substrate $K_{\mathrm{m}}[5,38]$, and (ii) coincidental, indirect activation resulting from off-target effects $[42,43]$. Skepticism about the direct mechanism arose from questions about the assay used. The initial screen for STACs that identified resveratrol used a fluorometric assay called "Fluor de Lys" [5] in which an acetylated peptide substrate was conjugated to aminomethylcoumarin (AMC) [5]. Separately, synthetic SIRT1 activators (e.g. SRT1460, SRT1720, and SRT2183) were discovered in a fluorescence polarization assay using a carboxytetramethylrhodamine (TAMRA)-tagged substrate and verified using mass spectrometry $[8,38,39]$. Numerous studies showed that STACs mimic the effect of SIRT1 activation in vivo on both the gene expression and physiological levels in organisms ranging from yeast to mice $[5,6,44]$. In dozens of studies, the effects of resveratrol and synthetic STACs were found to be Sir2/SIRT1-dependent [5, 6, $8,45]$. However, the legitimacy of STACs as direct SIRT1 activators was called into question by reports indicating that the fluorescent moieties on the substrates used in previous studies were required for SIRT1 activation [34, 40-42], leading some to conclude that SIRT1 activation was an in vitro artifact [42].

Since then, the direct mode of SIRT1 activation by STACs has received renewed support. The first report demonstrated that the fluorescent moiety on substrates is indeed dispensable for activation if replaced by hydrophobic amino acids [38]. This study also demonstrated that synthetic STACs physically interact with SIRT1 [38]. In a second study, native peptide substrates such as PGC-1a and FOXO3a were shown to mediate activation by resveratrol and synthetic STACs in vitro [39]. These substrates contain hydrophobic residues at the +1 and +6 positions relative to the acetylated lysine [39], the same positions as the fluorophores in the original assays $[5,8]$. These results demonstrated that there are structural and positional requirements in natural substrates that mediate SIRT1 activation. Using peptide microarrays, a separate study testing hundreds of substrates also showed that the effects of resveratrol on SIRT1 are substrate-selective. SIRT1 activation was observed with certain native sequences, while no effect or inhibition is observed with others [46]. These findings explain why no compound effects were previously found for some native substrates [40, 41].

Cumulatively, these data are consistent with several theories of SIRT1 activation. Steegborn and colleagues have proposed a model of direct interaction of the compound with enzymebound substrate [47], while Sauve, Sinclair and others have proposed an assisted-allosteric activation (AAA) mechanism in which STACs bind to a substrate-induced exosite on SIRT1 and in turn stabilize substrate binding and subsequent deacetylation [39] (Figure 2). In light of the recent discovery that SIRT6 can be activated by fatty acids via a similar $K_{m}$ lowering mechanism [48], a sirtuin with a short $\mathrm{N}$ terminus, the possibility that there are multiple modes of sirtuin activation must be entertained.

Additional clues to the SIRT1 activation mechanism have come from analysis of SIRT1 by genetic and biophysical approaches. A screen of random SIRT1 mutants identified a singlebase mutation in SIRT1 (SIRT1-E230K) that blocks both activation and STAC binding [39] (see Figure 2). Consistent with a common mechanism of activation for all $K_{\mathrm{m}}$-lowering STACs, the E230K substitution abrogated activation by all STACs tested, including 117 synthetic STACs from several distinct chemical classes. Furthermore, primary fibroblasts and myocytes reconstituted with SIRT1-E230K were unable to respond to the compounds, providing evidence that STACs can act directly on SIRT1 in vivo [39]. Interestingly, while the E230 residue is conserved from humans to flies, it is not present in the $\mathrm{N}$-terminal 
domains of worm or yeast Sir2 homologs, suggesting that other residues perform this function or that the activation dynamics for these homologs is different [39]. Further insights into the effects of STACs in vivo have come from studying their effects on aging and agerelated diseases, effects that typically mimic the overexpression of SIR2/SIRT1 (Figure 3).

\section{STACs in age-related disease}

\section{Aging}

Resveratrol and other related STACs extend the lifespan of numerous organisms including S. cerevisiae [5], C. elegans, D. melanogaster [1], N. furzeri, a short-lived fish, [49], and A. mellifera, the common honeybee [50]. In the first three instances, the longevity effects were shown to require Sir2 [1,5]. Consistent with the original sirtuin-CR hypothesis of Guarente [51], resveratrol and STACs induce physiological and gene expression changes consistent with CR, both in simple organisms and mammals [2, 52]. Resveratrol and STACs show promising effects in numerous age-related disease models including cancer, type 1 and 2 diabetes, inflammation, cardiovascular disease, stroke, hepatic steatosis, and others [8, 36]. Resveratrol supplementation prevents the development of cataracts and osteoporosis in mice during normal aging [36] and may delay infertility [53]. Several studies have reported that resveratrol does not extend the lifespan of mice when provided in a standard chow ad libitum $[4,54]$. However, resveratrol does extend mouse lifespan when provided in a high calorie diet or in standard chow fed to mice every other day [2, 54]. It has been proposed that while resveratrol appears to slow general age-related decline, it may not affect specific causes of death in certain mouse models (such as lymphoma), potentially explaining its differential effects on the lifespan of mice being fed a regular versus a high-fat diet [54]. SIRT1 may also have a greater effect on individuals that are under biological stress or lack homeostasis. The synthetic SIRT1 activator SRT1720 has also extended the lifespan of mice fed a high-calorie diet and protected against age-related changes in multiple tissues [45]. Whether or not synthetic STACs extend mouse lifespan on standard chow is not yet known.

\section{Cancer}

Considerable data exist on the role of SIRT1 and the effects of resveratrol in cancer [36]. However, like SIRT1, which has been described as both a tumor suppressor and a tumor promoter [55], the role of resveratrol in cancer treatment remains somewhat controversial. In 1997, a landmark study showed that topical application of resveratrol is chemopreventative in a model of skin cancer [36]. A further study demonstrated that the protective effects of resveratrol on cancer are mediated, in part, by SIRT1 [56]. Resveratrol slows the growth of numerous other cancers including colon [57], prostate, and lymphoma [58]. In contrast, Resveratrol has proven ineffective in breast cancer [36], spontaneous tumor formation and cancer-related deaths in old mice [54]. These disparate observations may be due to differences in dosages used in the various studies and the bioavailability of resveratrol in different tissues [36]. Moreover, one potential cause for resveratrol's apparent lack of systemic anti-cancer activity could be its pharmacokinetic properties [59]. Future studies will need to carefully account for differences in resveratrol concentrations in vitro and in vivo. Studies examining the effects of synthetic STACs on cancer have also yielded mixed results. SRT1720 induces apoptosis of multiple myeloma cells in vivo [60] but it also promotes breast cancer metastasis [61]. Despite dozens of cell-based and mouse cancer models that STACs have been tested in, there is no consensus on which approach would prove best in humans. In the end, whether to test an inhibitor or an activator may be specific to the type and genetics of the cancer. 


\section{Inflammation}

One of the main functions of SIRT1 is its anti-inflammatory activity, mediated in large part by inhibition of NF- $\kappa \mathrm{B}$, a key regulator of immune response [6]. This has led to considerable excitement about the use of STACs for the treatment of inflammatory and autoimmune disorders [36]. Resveratrol reduces levels of inflammatory cytokines in LPSstimulated macrophages [62] and peripheral blood mononuclear cells [63], protects cartilage against experimentally-induced inflammatory arthritis in rabbits [64], and inhibits inflammation caused by Listeria monocytogenes, a pathogen that typically affects immunocompromised individuals [65]. Other mouse models of tissue inflammation have also responded well to resveratrol, including chronic obstructive pulmonary disease (COPD) [66], and Crohn's disease [67]. Also, a recent study found that resveratrol exerts antiinflammatory effects in visceral white adipose tissue of rhesus monkeys fed a high-fat, highsugar diet [68]. Similar to resveratrol, the synthetic STAC SRT1720 has beneficial effects in mouse models of COPD/emphysema [69], and asthma [70]. It is worth noting that not all of the anti-inflammatory effects of resveratrol are mediated through SIRT1. For example, resveratrol is a direct non-selective pharmacological inhibitor of the pro-inflammatory cyclooxygenase enzymes COX1/2, the same enzymes targeted by aspirin [36].

\section{Cardiovascular disease}

The cardioprotective properties of resveratrol operate via multiple mechanisms, some of which are known to be mediated by SIRT1 [36]. The ability of resveratrol to scavenge free radicals and chelate copper reduces the oxidation of low-density lipoprotein (LDL) particles, a contributing factor to the development of coronary heart disease [36]. Resveratrol also decreases ROS levels, reduces lipid peroxidation, inhibits platelet aggregation, acts as a vasodilator, increases expression of endothelial and inducible nitric oxide synthase (eNOS and iNOS), and inhibits vascular smooth muscle cell (VSMC) proliferation [36, 71]. Effects of resveratrol on VSMC cells have recently been confirmed in monkeys [72]. Finally, resveratrol has been shown in vitro to block the adhesion of monocytes and granulocytes to endothelial cells by inhibiting expression of vascular cell adhesion molecule (VCAM) [73]. Similarly, SRT1720 has been shown to reduce myocardial infarction in mice [74], reduce whole-body fat mass and raise serum high density lipoprotein (HDL) levels, changes that positively associate with cardiovascular health[45].

\section{Neurodegeneration}

Resveratrol is able to cross the blood-brain barrier, where it can protect against stroke and brain damage, prevent cognitive decline, and promote general neuroprotection [36]. Both learning and memory are improved in aged mice subject to intraventricular injection of resveratrol for one week [75]. Perhaps even more impressive is the ability of resveratrol to attenuate symptoms of age-related neurological diseases such as Alzheimer's [76], Parkinson's disease [77], and multiple sclerosis [78]. Resveratrol slows the accumulation of amyloid-beta peptide, a causative agent in Alzheimer's disease [79] and dramatically reduces plaque formation in several regions of the brain [80]. Numerous studies are consistent with SIRT1 being the target of these effects. For example, neuroprotection by resveratrol requires SIRT1 [76], SRT1720 is protective in a model of multiple sclerosis [78], and SRT3025 (a blood-brain penetrant STAC) mimics the effects of CR on the brain and protects against neurodegeneration [81].

\section{Obesity and metabolism}

One of the best-studied effects of STACs is their ability to prevent and reverse the effects of obesity and age-related metabolic decline [45]. In mice fed a high calorie diet, resveratrol improves metabolism, protects against obesity, insulin resistance, and premature death $[2$, 
36]. Additionally, it has been reported that resveratrol prevents the formation of a fatty liver and induces genes for mitochondrial function, such as PGC-1a, a primary regulator of mitochondrial biogenesis $[2,36]$. However, this latter effect on PGC-1a may be confined to specific contexts as a recent report failed to observe any effects of resveratrol supplementation on mitochondrial biogenesis in rat muscle [82]. Several studies have also noted a remarkable ability for resveratrol to allay secondary phenotypes associated with diabetes such as diabetic nephropathy and tissue inflammation [83]. Similar to resveratrol, SRT1720 improves whole-body glucose homeostasis and insulin sensitivity, increases mitochondrial capacity [8], and decreases expression of oxidative stress markers and lysogenic enzymes [84]. Furthermore, SRT1720 extends both the mean and maximum lifespan of adult mice fed a high-fat diet, and improves mitochondrial bioenergetics in a SIRT1-dependent manner [45]. SRT2104 was reported to be well tolerated in humans, and to ameliorate the lipid profile of cigarette smokers [85].

In mice fed normal chow diets, resveratrol increases mitochondrial mass in muscle and liver, an effect that is due to SIRT1-mediated activation of LKB1 and subsequent activation of AMPK [32]. Consistent with this, several reports have described a large increase in exercise endurance and muscle fiber type shift to red or oxidative fibers in mice dosed with resveratrol [32]. Resveratrol has also enhances metabolism in non-human primates [86], and improves adipose insulin signaling in monkeys fed a high-fat, high-sugar diet [68]. In humans, resveratrol has had mixed results. SRT501, a proprietary formulation of resveratrol, improved glucose tolerance in type 2 diabetics in the absence of any adverse side-effects [87]. This study was supported by later work in humans describing an insulin-sensitizing effect of resveratrol [87]. Resveratrol supplementation also decreased the oxidative stress and inflammation caused by consumption of a high calorie meal [88]. More recently, 30-day resveratrol supplementation was shown to induce calorie restriction-like effects on energy metabolism and metabolic profile in obese humans [89]. However, a separate study demonstrated that resveratrol does not improve metabolic function in non-obese women with normal glucose tolerance [90]. The reason for the differences between these studies is not clear but it may be that STACs work to restore homeostasis in metabolically compromised individuals, and less so on healthy individuals, a possibility consistent with known functions of SIRT1 in animal studies. Clearly, additional clinical studies for resveratrol and synthetic STACs are warranted.

\section{Concluding remarks}

Only 10 years ago, the idea that a drug could treat one disease and prevent a dozen others was considered fanciful by many scientists. Today, however, with abundant data showing that multiple diseases of aging can be prevented (and in some cases reversed) by small molecules in rodents [2] and non-rodent primates [86], this possibility seems within reach. Despite progress, many key questions remain. For example, which phenotypes are due to the action of resveratrol and STACs directly on SIRT1, and which are due to effects on other targets? How does E230K mediate activation by STACs? Do the various alleles of SIRT1 in humans alter its substrate specificity or ability to be activated? Are there endogenous activators that bind to the same site as STACs?

A key question is how to test STACs in humans to give them the best chance of reaching patients. Regulatory agencies do not recognize aging as a disease, nor would a clinical trial to slow aging be cost-effective. If synthetic STACs are to benefit patients, they almost certainly will be approved and prescribed as medicines for specific diseases. Animal studies show that STACs may be useful for treating diseases of aging, but they may have additional health benefits such as protection against viral infections [36]. Resveratrol and the more potent synthetic STACs are in clinical trials that will provide critical information about the 
safety and efficacy of SIRT1 activation (Box 3). Positive data from these trials indicate that STACs can effectively treat metabolic and inflammatory diseases in patients.

But there is still a long road ahead. Making an approved drug is a serious challenge, arguably more difficult (and certainly more expensive) than the work done to date. A single medicine can cost upwards of $\sim$ \$US 1 billion and takes many years to develop.

Governmental regulatory authorities do not recognize aging as a disease, largely because it is too common, so the first uses of STACs (and other novel anti-aging molecules) will need to be developed for individual diseases. Knowing these challenges, millions of people have chosen not to wait for an approved drug, taking a daily STAC in the form of resveratrol, partaking in one of the largest uncontrolled experiments in the history of aging research, a situation that only increases the importance and urgency of studying SIRT1 activation.

\section{References}

1. Wood JG, et al. Sirtuin activators mimic caloric restriction and delay ageing in metazoans. Nature. 2004; 430:686-689. [PubMed: 15254550]

2. Baur JA, et al. Resveratrol improves health and survival of mice on a high-calorie diet. Nature. 2006; 444:337-342. [PubMed: 17086191]

3. Harrison DE, et al. Rapamycin fed late in life extends lifespan in genetically heterogeneous mice. Nature. 2009; 460:392-395. [PubMed: 19587680]

4. Strong R, et al. Evaluation of resveratrol, green tea extract, curcumin, oxaloacetic acid, and medium-chain triglyceride oil on life span of genetically heterogeneous mice. J Gerontol A Biol Sci Med Sci. 2013; 68:6-16. [PubMed: 22451473]

5. Howitz KT, et al. Small molecule activators of sirtuins extend Saccharomyces cerevisiae lifespan. Nature. 2003; 425:191-196. [PubMed: 12939617]

6. Morris BJ. Seven sirtuins for seven deadly diseases of aging. Free Radic Biol Med. 2013; 56:133171. [PubMed: 23104101]

7. Kenyon C. The first long-lived mutants: discovery of the insulin/IGF-1 pathway for ageing. Philos Trans R Soc Lond B Biol Sci. 2011; 366:9-16. [PubMed: 21115525]

8. Milne JC, et al. Small molecule activators of SIRT1 as therapeutics for the treatment of type 2 diabetes. Nature. 2007; 450:712-716. [PubMed: 18046409]

9. Haigis MC, Guarente LP. Mammalian sirtuins--emerging roles in physiology, aging, and calorie restriction. Genes Dev. 2006; 20:2913-2921. [PubMed: 17079682]

10. Bordone L, Guarente L. Calorie restriction, SIRT1 and metabolism: understanding longevity. Nat Rev Mol Cell Biol. 2005; 6:298-305. [PubMed: 15768047]

11. Blander G, Guarente L. The Sir2 family of protein deacetylases. Annu Rev Biochem. 2004; 73:417-435. [PubMed: 15189148]

12. Tissenbaum HA, Guarente L. Increased dosage of a sir-2 gene extends lifespan in Caenorhabditis elegans. Nature. 2001; 410:227-230. [PubMed: 11242085]

13. Rogina B, Helfand SL. Sir2 mediates longevity in the fly through a pathway related to calorie restriction. Proc Natl Acad Sci U S A. 2004; 101:15998-16003. [PubMed: 15520384]

14. Burnett C, et al. Absence of effects of Sir2 overexpression on lifespan in C. elegans and Drosophila. Nature. 2011; 477:482-485. [PubMed: 21938067]

15. Viswanathan M, Guarente L. Regulation of Caenorhabditis elegans lifespan by sir-2.1 transgenes. Nature. 2011; 477:E1-2. [PubMed: 21938026]

16. Banerjee KK, et al. dSir2 in the adult fat body, but not in muscles, regulates life span in a dietdependent manner. Cell Rep. 2012; 2:1485-1491. [PubMed: 23246004]

17. Kanfi Y, et al. The sirtuin SIRT6 regulates lifespan in male mice. Nature. 2012; 483:218-221. [PubMed: 22367546]

18. Satoh A, et al. Sirt1 Extends Life Span and Delays Aging in Mice through the Regulation of Nk2 Homeobox 1 in the DMH and LH. Cell Metab. 2013; 18:416-430. [PubMed: 24011076] 
19. Bitterman KJ, et al. Inhibition of silencing and accelerated aging by nicotinamide, a putative negative regulator of yeast sir2 and human SIRT1. J Biol Chem. 2002; 277:45099-45107. [PubMed: 12297502]

20. Imai S, et al. Transcriptional silencing and longevity protein Sir2 is an NAD-dependent histone deacetylase. Nature. 2000; 403:795-800. [PubMed: 10693811]

21. Jiang H, et al. SIRT6 regulates TNF-alpha secretion through hydrolysis of long-chain fatty acyl lysine. Nature. 2013; 496:110-113. [PubMed: 23552949]

22. Feldman JL, et al. Activation of the Protein Deacetylase SIRT6 by Long-chain Fatty Acids and Widespread Deacylation by Mammalian Sirtuins. J Biol Chem. 2013

23. Anderson RM, et al. Nicotinamide and PNC1 govern lifespan extension by calorie restriction in Saccharomyces cerevisiae. Nature. 2003; 423:181-185. [PubMed: 12736687]

24. Lin SJ, et al. Requirement of NAD and SIR2 for life-span extension by calorie restriction in Saccharomyces cerevisiae. Science. 2000; 289:2126-2128. [PubMed: 11000115]

25. Lin SJ, et al. Calorie restriction extends Saccharomyces cerevisiae lifespan by increasing respiration. Nature. 2002; 418:344-348. [PubMed: 12124627]

26. Greer EL, Brunet A. Different dietary restriction regimens extend lifespan by both independent and overlapping genetic pathways in C. elegans. Aging Cell. 2009; 8:113-127. [PubMed: 19239417]

27. Wang Y, Tissenbaum HA. Overlapping and distinct functions for a Caenorhabditis elegans SIR2 and DAF-16/FOXO. Mech Ageing Dev. 2006; 127:48-56. [PubMed: 16280150]

28. Raynes R, et al. Heat shock and caloric restriction have a synergistic effect on the heat shock response in a sir2.1-dependent manner in Caenorhabditis elegans. J Biol Chem. 2012; 287:2904529053. [PubMed: 22778258]

29. Cohen HY, et al. Calorie restriction promotes mammalian cell survival by inducing the SIRT1 deacetylase. Science. 2004; 305:390-392. [PubMed: 15205477]

30. Bordone L, et al. SIRT1 transgenic mice show phenotypes resembling calorie restriction. Aging Cell. 2007; 6:759-767. [PubMed: 17877786]

31. Chen D, et al. Tissue-specific regulation of SIRT1 by calorie restriction. Genes Dev. 2008; 22:1753-1757. [PubMed: 18550784]

32. Price NL, et al. SIRT1 is required for AMPK activation and the beneficial effects of resveratrol on mitochondrial function. Cell Metab. 2012; 15:675-690. [PubMed: 22560220]

33. Biason-Lauber A, et al. Identification of a SIRT1 mutation in a family with type 1 diabetes. Cell Metab. 2013; 17:448-455. [PubMed: 23473037]

34. Blum CA, et al. SIRT1 modulation as a novel approach to the treatment of diseases of aging. J Med Chem. 2011; 54:417-432. [PubMed: 21080630]

35. Sauve AA, et al. Chemical activation of Sir2-dependent silencing by relief of nicotinamide inhibition. Mol Cell. 2005; 17:595-601. [PubMed: 15721262]

36. Baur JA, Sinclair DA. Therapeutic potential of resveratrol: the in vivo evidence. Nat Rev Drug Discov. 2006; 5:493-506. [PubMed: 16732220]

37. Lamuela-Raventos R, et al. Direct HPLC Analysis of cis- and trans-Resveratrol and Piceid Isomers in Spanish Red Vitis vinifera Wines. Journal of Agricultural and Food Chemistry. 1995; 43:281283.

38. Dai H, et al. SIRT1 activation by small molecules: kinetic and biophysical evidence for direct interaction of enzyme and activator. J Biol Chem. 2010; 285:32695-32703. [PubMed: 20702418]

39. Hubbard BP, et al. Evidence for a common mechanism of SIRT1 regulation by allosteric activators. Science. 2013; 339:1216-1219. [PubMed: 23471411]

40. Borra MT, et al. Mechanism of human SIRT1 activation by resveratrol. J Biol Chem. 2005; 280:17187-17195. [PubMed: 15749705]

41. Kaeberlein M, et al. Substrate-specific activation of sirtuins by resveratrol. J Biol Chem. 2005; 280:17038-17045. [PubMed: 15684413]

42. Pacholec M, et al. SRT1720, SRT2183, SRT1460, and resveratrol are not direct activators of SIRT1. J Biol Chem. 2010; 285:8340-8351. [PubMed: 20061378]

43. Park SJ, et al. Resveratrol ameliorates aging-related metabolic phenotypes by inhibiting cAMP phosphodiesterases. Cell. 2012; 148:421-433. [PubMed: 22304913] 
44. Smith JJ, et al. Small molecule activators of SIRT1 replicate signaling pathways triggered by calorie restriction in vivo. BMC Syst Biol. 2009; 3:31. [PubMed: 19284563]

45. Minor RK, et al. SRT1720 improves survival and healthspan of obese mice. Sci Rep. 2011; 1:70. [PubMed: 22355589]

46. Lakshminarasimhan M, et al. Sirt1 activation by resveratrol is substrate sequence-selective. Aging (Albany NY). 2013; 5:151-154. [PubMed: 23524286]

47. Gertz M, et al. A molecular mechanism for direct sirtuin activation by resveratrol. PLoS One. 2012; 7:e49761. [PubMed: 23185430]

48. Feldman JL, et al. Activation of the Protein Deacetylase SIRT6 by Long-chain Fatty Acids and Widespread Deacylation by Mammalian Sirtuins. J Biol Chem. 2013; 288:31350-31356. [PubMed: 24052263]

49. Valenzano DR, et al. Resveratrol prolongs lifespan and retards the onset of age-related markers in a short-lived vertebrate. Curr Biol. 2006; 16:296-300. [PubMed: 16461283]

50. Rascon B, et al. The lifespan extension effects of resveratrol are conserved in the honey bee and may be driven by a mechanism related to caloric restriction. Aging (Albany NY). 2012; 4:499_ 508. [PubMed: 22868943]

51. Guarente L. Sir2 links chromatin silencing, metabolism, and aging. Genes Dev. 2000; 14:10211026. [PubMed: 10809662]

52. Barger JL, et al. A low dose of dietary resveratrol partially mimics caloric restriction and retards aging parameters in mice. PLoS One. 2008; 3:e2264. [PubMed: 18523577]

53. Liu M, et al. Resveratrol protects against age-associated infertility in mice. Hum Reprod. 2013; 28:707-717. [PubMed: 23293221]

54. Pearson KJ, et al. Resveratrol delays age-related deterioration and mimics transcriptional aspects of dietary restriction without extending life span. Cell Metab. 2008; 8:157-168. [PubMed: 18599363]

55. Deng CX. SIRT1, is it a tumor promoter or tumor suppressor? Int J Biol Sci. 2009; 5:147-152. [PubMed: 19173036]

56. Boily G, et al. SirT1-null mice develop tumors at normal rates but are poorly protected by resveratrol. Oncogene. 2009; 28:2882-2893. [PubMed: 19503100]

57. Firestein R, et al. The SIRT1 deacetylase suppresses intestinal tumorigenesis and colon cancer growth. PLoS One. 2008; 3:e2020. [PubMed: 18414679]

58. Aggarwal BB, et al. Role of resveratrol in prevention and therapy of cancer: preclinical and clinical studies. Anticancer Res. 2004; 24:2783-2840. [PubMed: 15517885]

59. Boocock DJ, et al. Phase I dose escalation pharmacokinetic study in healthy volunteers of resveratrol, a potential cancer chemopreventive agent. Cancer Epidemiol Biomarkers Prev. 2007; 16:1246-1252. [PubMed: 17548692]

60. Chauhan D, et al. Preclinical evaluation of a novel SIRT1 modulator SRT1720 in multiple myeloma cells. Br J Haematol. 2011; 155:588-598. [PubMed: 21950728]

61. Suzuki K, et al. SRT1720, a SIRT1 activator, promotes tumor cell migration, and lung metastasis of breast cancer in mice. Oncol Rep. 2012; 27:1726-1732. [PubMed: 22470132]

62. Qureshi AA, et al. Inhibition of nitric oxide and inflammatory cytokines in LPS-stimulated murine macrophages by resveratrol, a potent proteasome inhibitor. Lipids Health Dis. 2012; 11:76. [PubMed: 22698256]

63. Lanzilli G, et al. Anti-inflammatory effect of resveratrol and polydatin by in vitro IL-17 modulation. Inflammation. 2012; 35:240-248. [PubMed: 21369944]

64. Elmali N, et al. Effects of resveratrol in inflammatory arthritis. Inflammation. 2007; 30:1-6. [PubMed: 17115116]

65. Park DW, et al. Resveratrol inhibits inflammation induced by heat-killed Listeria monocytogenes. J Med Food. 2012; 15:788-794. [PubMed: 22857612]

66. Zhou M, et al. Effect of resveratrol on chronic obstructive pulmonary disease in rats and its mechanism. Yao Xue Xue Bao. 2008; 43:128-132. [PubMed: 18507337]

67. Rahal K, et al. Resveratrol has antiinflammatory and antifibrotic effects in the peptidoglycanpolysaccharide rat model of Crohn's disease. Inflamm Bowel Dis. 2012; 18:613-623. [PubMed: 22431488] 
68. Jimenez-Gomez Y, et al. Resveratrol improves adipose insulin signaling and reduces the inflammatory response in adipose tissue of rhesus monkeys on high-fat, high-sugar diet. Cell Metab. 2013; 18:533-545. [PubMed: 24093677]

69. Yao H, et al. SIRT1 protects against emphysema via FOXO3-mediated reduction of premature senescence in mice. J Clin Invest. 2012; 122:2032-2045. [PubMed: 22546858]

70. Ichikawa T, et al. Sirtuin 1 activator SRT1720 suppresses inflammation in an ovalbumin-induced mouse model of asthma. Respirology. 2013; 18:332-339. [PubMed: 23062010]

71. Zheng JP, et al. Resveratrol induces p53 and suppresses myocardin-mediated vascular smooth muscle cell differentiation. Toxicol Lett. 2010; 199:115-122. [PubMed: 20797428]

72. Csiszar A, et al. Age-associated proinflammatory secretory phenotype in vascular smooth muscle cells from the non-human primate Macaca mulatta: reversal by resveratrol treatment. J Gerontol A Biol Sci Med Sci. 2012; 67:811-820. [PubMed: 22219513]

73. Ferrero ME, et al. Activity in vitro of resveratrol on granulocyte and monocyte adhesion to endothelium. Am J Clin Nutr. 1998; 68:1208-1214. [PubMed: 9846848]

74. Tong C, et al. Impaired SIRT1 nucleocytoplasmic shuttling in the senescent heart during ischemic stress. FASEB J. 2012

75. Zhao YN, et al. Resveratrol improves learning and memory in normally aged mice through microRNA-CREB pathway. Biochem Biophys Res Commun. 2013; 435:597-602. [PubMed: 23685142]

76. Kim D, et al. SIRT1 deacetylase protects against neurodegeneration in models for Alzheimer's disease and amyotrophic lateral sclerosis. EMBO J. 2007; 26:3169-3179. [PubMed: 17581637]

77. Lofrumento DD, et al. Neuroprotective effects of resveratrol in an MPTP mouse model of Parkinson's-like disease: Possible role of SOCS-1 in reducing pro-inflammatory responses. Innate Immun. 2013

78. Shindler KS, et al. Oral resveratrol reduces neuronal damage in a model of multiple sclerosis. J Neuroophthalmol. 2010; 30:328-339. [PubMed: 21107122]

79. Marambaud P, et al. Resveratrol promotes clearance of Alzheimer's disease amyloid-beta peptides. J Biol Chem. 2005; 280:37377-37382. [PubMed: 16162502]

80. Karuppagounder SS, et al. Dietary supplementation with resveratrol reduces plaque pathology in a transgenic model of Alzheimer's disease. Neurochem Int. 2009; 54:111-118. [PubMed: 19041676]

81. Graff J, et al. A Dietary Regimen of Caloric Restriction or Pharmacological Activation of SIRT1 to Delay the Onset of Neurodegeneration. J Neurosci. 2013; 33:8951-8960. [PubMed: 23699506]

82. Higashida K, et al. Effects of resveratrol and SIRT1 on PGC-1alpha activity and mitochondrial biogenesis: a reevaluation. PLoS Biol. 2013; 11:e1001603. [PubMed: 23874150]

83. Jiang B, et al. Resveratrol attenuates early diabetic nephropathy by down-regulating glutathione stransferases mu in diabetic rats. J Med Food. 2013; 16:481-486. [PubMed: 23767859]

84. Yamazaki Y, et al. Treatment with SRT1720, a SIRT1 activator, ameliorates fatty liver with reduced expression of lipogenic enzymes in MSG mice. Am J Physiol Endocrinol Metab. 2009; 297:E1179-1186. [PubMed: 19724016]

85. Venkatasubramanian S, et al. Cardiovascular Effects of a Novel SIRT1 Activator, SRT2104, in Otherwise Healthy Cigarette Smokers. J Am Heart Assoc. 2013; 2:e00042. [PubMed: 23770971]

86. Fiori JL, et al. Resveratrol Prevents beta-cell Dedifferentiation in Non-Human Primates Given a High Fat/ High Sugar Diet. Diabetes. 2013

87. Smoliga JM, et al. Resveratrol and health--a comprehensive review of human clinical trials. Mol Nutr Food Res. 2011; 55:1129-1141. [PubMed: 21688389]

88. Ghanim H, et al. A resveratrol and polyphenol preparation suppresses oxidative and inflammatory stress response to a high-fat, high-carbohydrate meal. J Clin Endocrinol Metab. 2011; 96:14091414. [PubMed: 21289251]

89. Timmers S, et al. Calorie restriction-like effects of 30 days of resveratrol supplementation on energy metabolism and metabolic profile in obese humans. Cell Metab. 2011; 14:612-622. [PubMed: 22055504] 
90. Yoshino J, et al. Resveratrol supplementation does not improve metabolic function in nonobese women with normal glucose tolerance. Cell Metab. 2012; 16:658-664. [PubMed: 23102619]

91. Avalos JL, et al. Mechanism of sirtuin inhibition by nicotinamide: altering the NAD $(+)$ cosubstrate specificity of a Sir2 enzyme. Mol Cell. 2005; 17:855-868. [PubMed: 15780941]

92. Zhao K, et al. Structural basis for nicotinamide cleavage and ADP-ribose transfer by NAD(+)dependent Sir2 histone/protein deacetylases. Proc Natl Acad Sci U S A. 2004; 101:8563-8568. [PubMed: 15150415]

93. Smith BC, Denu JM. Mechanism-based inhibition of Sir2 deacetylases by thioacetyl-lysine peptide. Biochemistry. 2007; 46:14478-14486. [PubMed: 18027980]

94. Asaba T, et al. Inhibition of human sirtuins by in situ generation of an acetylated lysine-ADPribose conjugate. J Am Chem Soc. 2009; 131:6989-6996. [PubMed: 19413317]

95. Villalba JM, Alcain FJ. Sirtuin activators and inhibitors. Biofactors. 2012; 38:349-359. [PubMed: 22730114]

96. Medda F, et al. Novel cambinol analogs as sirtuin inhibitors: synthesis, biological evaluation, and rationalization of activity. J Med Chem. 2009; 52:2673-2682. [PubMed: 19419202]

97. Rotili D, et al. Identification of tri- and tetracyclic pyrimidinediones as sirtuin inhibitors. ChemMedChem. 2010; 5:674-677. [PubMed: 20391556]

98. Solomon JM, et al. Inhibition of SIRT1 catalytic activity increases p53 acetylation but does not alter cell survival following DNA damage. Mol Cell Biol. 2006; 26:28-38. [PubMed: 16354677]

99. Gertz M, et al. Ex-527 inhibits Sirtuins by exploiting their unique NAD+-dependent deacetylation mechanism. Proc Natl Acad Sci U S A. 2013; 110:E2772-2781. [PubMed: 23840057]

100. Zhang Y, et al. Deacetylation of cortactin by SIRT1 promotes cell migration. Oncogene. 2009; 28:445-460. [PubMed: 18850005]

101. Zhou X, et al. Sirtuin 1 inhibition delays cyst formation in autosomal-dominant polycystic kidney disease. J Clin Invest. 2013

102. Gresele P, et al. Resveratrol, at concentrations attainable with moderate wine consumption, stimulates human platelet nitric oxide production. J Nutr. 2008; 138:1602-1608. [PubMed: 18716157]

103. Elliott PJ, Jirousek M. Sirtuins: novel targets for metabolic disease. Curr Opin Investig Drugs. 2008; 9:371-378.

104. Howells LM, et al. Phase I randomized, double-blind pilot study of micronized resveratrol (SRT501) in patients with hepatic metastases--safety, pharmacokinetics, and pharmacodynamics. Cancer Prev Res (Phila). 2011; 4:1419-1425. [PubMed: 21680702]

105. Hoffmann E, et al. Pharmacokinetics and tolerability of SRT2104, a first-in-class small molecule activator of SIRT1, after single and repeated oral administration in man. Br J Clin Pharmacol. 2013; 75:186-196. [PubMed: 22616762]

106. Libri V, et al. A pilot randomized, placebo controlled, double blind phase I trial of the novel SIRT1 activator SRT2104 in elderly volunteers. PLoS One. 2012; 7:e51395. [PubMed: 23284689] 


\section{Box 3}

\section{Pharmacokinetics, safety and toxicity of STACs}

Human studies show a dose-dependent relationship exists between resveratrol intake and its maximum plasma concentration $\left(\mathrm{C}_{\max }\right)$ [87]. Two human studies involving supplementation with $5 \mathrm{~g}$ of trans-resveratrol reported a 24 hour mean plasma concentration of $\sim 52 \mu \mathrm{g} / \mathrm{L}$ resveratrol over a one day period, with a $\mathrm{C}_{\max }$ value of 540 $\mu \mathrm{g} / \mathrm{L}$ occurring 1.5 hours after administration [87]. Likewise, a separate human study involving dietary supplementation with $1 \mathrm{~g}$ of resveratrol reported plasma concentrations ranging from 8.3 to $404 \mu \mathrm{g} / \mathrm{L}$ one hour following ingestion [87]. Multiple or daily resveratrol dosing seems to augment these figures slightly [87]. Importantly, these findings are in agreement with multiple studies in animal models suggesting that the effectiveness of resveratrol and similar polyphenols may be limited by poor systemic bioavailability, and rapid metabolism [2]. A total of five distinct metabolites of resveratrol have been reported in urine samples, with a half life of $\sim 9.2$ hours [36]. Indeed, one study has approximated peak levels of two resveratrol metabolites, monoglucuronides and resveratrol-3-sulfate, to be substantially higher in the blood than the unmodified molecule [36]. This observation led some researchers to surmise that some of the effects of resveratrol treatment result from its modified forms, a hypothesis supported by preliminary studies [2]. Given the limited bioavailability of resveratrol, its derivative formulations and synthetic SIRT1 activators offer more promise for exploiting the potential benefits of SIRT1. A formulation of micronized resveratrol, SRT501, achieved blood levels 5-8 times higher than trans-resveratrol alone, concentrations that approach levels that have shown efficacy in animal models [103, 104]. Repeated oral administration of SRT2104 in humans displayed good tolerability and reasonable bioavailability ( $14 \%$ with mean clearance $400 \mathrm{~mL} / \mathrm{min}$ ), which was enhanced with food [105]. No toxicity effects of SRT2104 were observed in phase I clinical trials following administration to elderly volunteers [106]. 


\section{Highlights}

- Sirtuins (SIRT1-7) are $\mathrm{NAD}^{+}$-dependent deacylases than promote homeostasis.

- SIRT1 activation shows promise for the treatment of aging and age-related diseases.

- Natural and synthetic SIRT1 activators (STACs) have been discovered.

- There is a common SIRT1 activation mechanism mediated by an $\mathrm{N}$-terminal domain.

- Clinical trials indicate that STACs are efficacious but questions remain. 


\section{A First generation STACs (2003)}

Resveratrol<smiles>Oc1ccc(/C=C/c2cc(O)cc(O)c2)cc1</smiles>

Quercetin<smiles>O=c1c(O)c(-c2ccc(O)c(O)c2)oc2cc(O)cc(O)c12</smiles>

Butein<smiles>O=C(/C=C/c1ccc(O)c(O)c1)c1ccc(O)cc1O</smiles>

B Second generation STACs (2007)

C Third Generation STACs (2013)

STAC-5

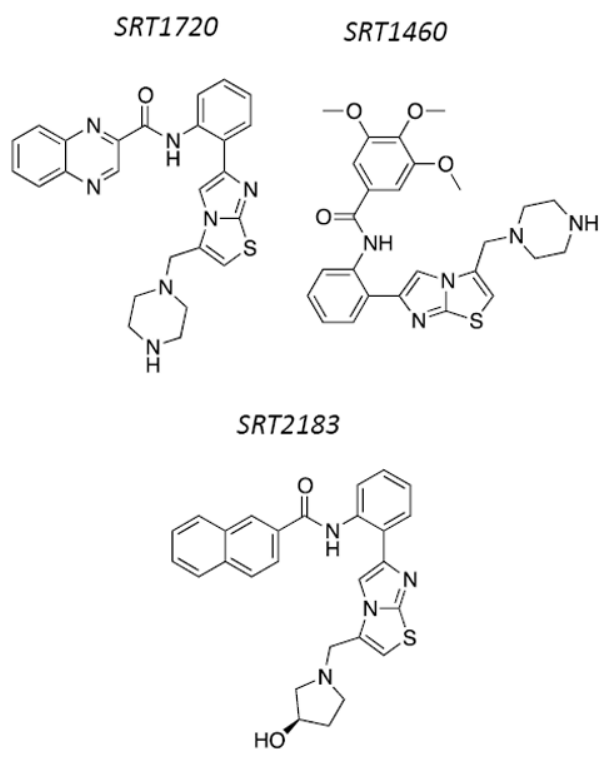<smiles>O=C(Nc1nccs1)c1cncc2[nH]c(-c3ccccc3C(F)(F)F)cc12</smiles><smiles>O=C(Nc1ccncc1)N1CCc2ccc(-c3cccc(C(F)(F)F)c3)nc21</smiles><smiles>O=C([Se])[CH]C(=O)N1CCCOc2ccc(-c3cccc(Cl)c3)nc21</smiles>

Figure 1. Sirtuin activating compounds (STACs) that extend lifespan and/or healthspan The quest to find molecules that slow aging has rapidly advanced in recent years. Small molecule SIRT1 activators include (A) First generation molecules such as resveratrol and like polyphenols [5], (B) second generation STACs such as the imidazothiazoles [8], and (C) third generation STACs such as benzimidazoles and urea-based scaffolds [38, 39]. 
Non-productive STAC binding
or no binding

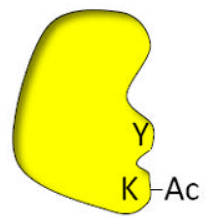

$\mathrm{NH}_{2}$

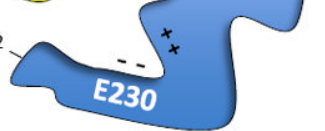

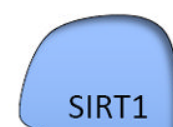

\section{SIRT1}

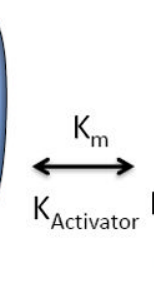

Productive drug-binding in induced exosite
Release of deacetylated substrate, products, and STAC

Figure 2. Proposed mechanism for allosteric SIRT1 activation by STACs

SIRT1 deacetylates target proteins (yellow) using $\mathrm{NAD}^{+}$as a cosubstrate to generate a deacetylated product, O-acetyl-ADP ribose (OAcADPR) and nicotinamide (NAM).

Biochemical and structural data favor a mechanism of direct "assisted allosteric activation" by STACs that is mediated by an N-terminal activation domain. Mutation of a conserved glutamate to lysine or alanine (E230K/A) in a structured $\mathrm{N}$-terminal domain blocks activation by resveratrol and 117 synthetic STACs, arguing for a common mechanism of activation [39]. Fluorophores that were initially thought to be a requisite for activation in vitro are now known to mimic natural hydrophobic amino acids adjacent to the acetylated lysine (denoted by a Y in substrate). Binding of substrates with hydrophobic motifs near the acetyl site induce a conformational change upon binding, forming a specific exosite that allows activators to bind in a productive manner and in turn stabilize the docked substrate. Positive charges C-terminal to E230K interact with the negative charge of E230 to assist with the enzyme-STAC-substrate complex. Whether or not peptide $K_{\mathrm{m}}$ corresponds to $K_{\mathrm{d}}$ is not yet known. 


\section{Physiological Effects of STACs}

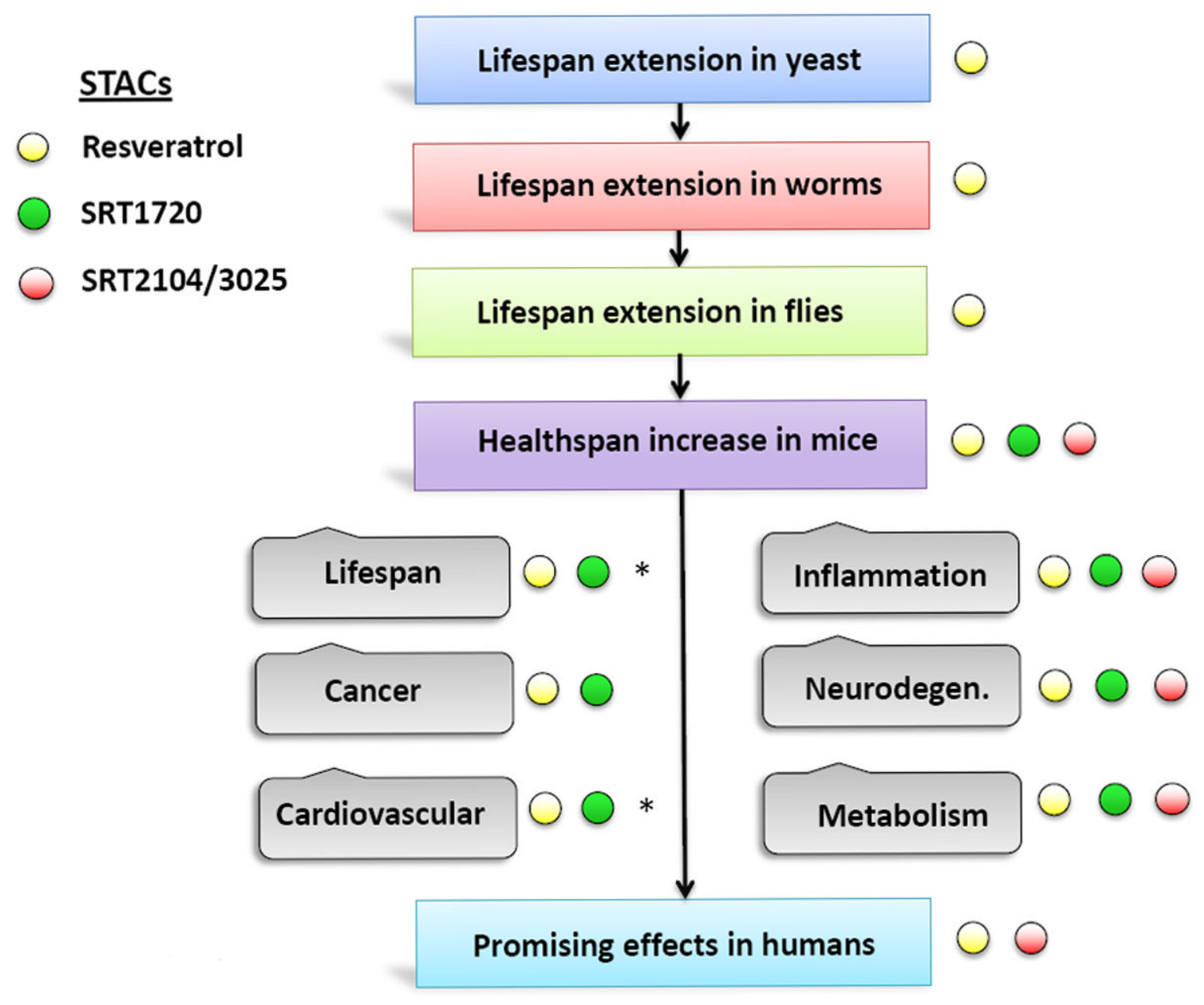

Figure 3. Outline of STAC effects on aging and age-related diseases

Resveratrol - yellow peg, SRT1720 - green peg, SRT2104 and the related brain-penetrant molecule SRT3025 - red peg. Age-related diseases on the right panel that are ameliorated by STACs are indicated with colored peg. Asterisks denote studies in progress. 